\title{
Article \\ Soil and Crop/Tree Segmentation from Remotely Sensed Data by Using Digital Surface Models
}

\author{
Adriano Mancini ${ }^{\dagger}$, Jack Dyson ${ }^{\dagger}$, Emanuele Frontoni ${ }^{\dagger *}$ and Primo Zingaretti ${ }^{\dagger}$ \\ * Correspondence: e.frontoni@univpm.it \\ † Dipartimento di Ingegneria dell'Informazione, Università Politecnica delle Marche, Ancona, ITALY
}

\begin{abstract}
The increased availability of high resolution remote sensor data for precision agriculture applications permits users to aquire deeper and more relevant knowledge about crops states that lead inevitably to better decisions. The algorithm libraries being developed and evolved around these applications rely on multi-spectral or hyper-spectral data acquired by using manned or unmanned platforms. The current state of the art makes thorough use of vegetational indicies to guide the operational management of agricultural land plots. One of the most challenging sub-problems is to correctly identify and separate crop from soil. Thresholding techniques based on Normalized Difference Vegetation Index (NDVI) or other such similar metrics have the advantage of being simple, easy to read transformations of the data packed with useful information. Obvious difficulties arise when crop/tree and soil have similar spectral responses as in case of grass filled areas in vineyards. In this case grass and canopy are close in terms of NDVI values and thresholding techniques will generally fail. Radiometric approaches could be integrated or replaced by a geometric approach that is based on terrain data like Digital Surface Models (DSMs). These models are one of the ouputs of orthorectification engines usually present in data acquired by using unmanned platforms. In this paper we present two approaches based on DSM that are able to segment crop/tree from soil while over gradient terrain. The DSM data are processed through a two dimensional data slicing or reduction technique. Each slice is separately processed as a one dimensional time series to derive the terrain and tree structures separately, here interpreted as object probability densities. In particular the first approach is a Cartesian grid rasterization (CARSCAN) of the terrain and the second is its immediate generalisation or radial grid rasterization of the DSM model (FANSCAN). The FANSCAN recovers information from the original image at greater frequencies on the Fourier plane. These approaches enable the identification of crop/tree from soil in case of slopes or hilly terrain without any constraint on the displacement / direction of plant/tree row. The proposed algorithm uses pure DSM information even if it is possible to fuse its output with other classifiers.
\end{abstract}

Keywords: Segmentation; multi-spectral camera; soil: tree; raster scanning; UAV application

\section{Introduction}

The acquisition of high resolution imagery for precision agriculture applications is a common task for a large variety of users as agronomists, big-data specialists and researchers. Unmanned system are able to capture data with ultra high resolution (up to $1 \mathrm{~cm}$ of terrain) also by using multi-spectral or hyper-spectral payloads. Typically data are acquired by a large set of overlapping images that are post-processed to derive a single global ortho-photo of the region of interest. The main advantages of such platforms is data aquisition in presence of cloud overlay which a satellite cannot do. On the contrary the cost of surveying can increase despite the availablility several low cost flight platforms and payloads [1]. In this new high-resolution era, due in part to Unmanned Aerial Systems (UASs) 
[2], opens new ways to analyze the fields, crops and trees during the growing process for proper management of all operations (e.g. applied, tilling,...) in order to maximize yield, quality and optimize costs [3]. In this sense, UAS platforms stand ready to overcome the main limitations of satellite platforms, ensuring very high resolution spectral, spatio-temporal data aquisition systems [4]. In this scenario, the data play a key role in feature extraction where the manipulation of spectral bands is the classical methodological tool to start an analysis - possibly as an input feed to other methods for further analysis. Vegetational indices are usually used in a context where machine leaning algorithms are used to classify data in both pixel and object domains [5]. These become more effective if they are given access to a proper feature set at the start of their analysis runs. The planning of task (e.g., variable rate) requires a deep knowledge of crops and their status [6]. The classical output of an analysis from an expert is a prescription map that will map tractor operations like spraying or treatment application over the field. The generation of a prescription map requires the definition of management zones that reflect areas and their status [7]. The typical case is variable rate Nitrogen fertilizer application as discussed in [8]. The generation of management zones and their prescription maps may then be automated starting from decision support systems that fuse heterogeneous data as well the soil signal and the previous yield together with the vegetation indices $[9,10]$.

When performing an analysis based on vegetation indices, it is important to consider only data relevant to the problem. Here by the term relevant we mean pixels related to crop or tree field without considering the soil variation. In this case the segmentation of soil and crop or tree field has a strong impact on the evaluation of region of interest. The segmentation process of crop and tree vs soil could be considered as an advanced Land Use or Land Cover mapping. The identification of crops could be carried out by using spectral or spatial or indeed both features. Spectral segmentation usually relies on supervised or unsupervised algorithms also including the use of satellite data [11]. One of the important requirements, as mentioned above, is that both soil and crops must have a different spectral signature. When GSD are of the order of $1-2$ meters a lot of ground noise is mapped into the pixels and the result is that the underlying soil response could be influenced by the crop sgnal just above it. In this case it is necessary to increase the resolution and UAS platforms are the suitable systems to gather these data.

High resolution images can cause further problems through the data intrinsic noise signals. An image with a 1 centimeter GSD is quite challenging to analyze considering the high variability of crop and soil signals. In this case other than pure spectral features, the spatial and geometric features become useful in order to extract further information about the ground truth probability distribution in the data. In particular, the crop height field is an important but simple mathematical variable to indicate crop vs soil signal rations. It can also act as a sensor able to measure crop's growth [12]. Volume estimation is also possible and this represents and additional variable to use in the decision making process [13]. Synthetic Aperture RADAR (SAR) can also be used to retrieve agricultural crop height from the event space even if the resolution and cost are challenging [14]. A viable solution is the use of UAS platforms that are able to measure height by direct and indirect methods. Such systems are able to host compact multi-spectral and hyper-spectral sensors [15] acquiring images that are orthorectified by using approach as Structure from Motion (SfM) that is a part of the overall processing pipeline [16]. The quantification and identification of soil and vegetation is important for several purposes [17] as an estimator for growth [18,19], 3D monitoring [20] and weed identification [21,22]. The identification of weeds is important to ensure uniform growth of the target crop [23] and is also supported by methods able to classify crops, weeds and their foundation soils [24] through the use of Excess Green Index (ExG) [25].

Vineyards and fruit plants are a typical example of complex regions in both detection and study. Slope in the terrain and also the presence of grassed soil substantially influence the overall terrain statistics. Detection can be carried out by using algorithms based on frequency analysis [26], Hough Space Clustering or Total Least Squares as in [27]. 


\begin{abstract}
In this paper we propose a novel method named FANSCAN that extend our previous methods [28] (also CARSCAN) to segment canopy/tree coverage vs the underlying soil. The segmented image is fundamental to correctly performing an analysis that requires the exact knowledge of the canopy position. FANSCAN is also related to our previous research to extract objects from complex data-set as the case of Lidar-Multispectral as described in [29,30]. Previous work proposed a slicing approach that fuses adaptive thresholding and 1D scan of the images. The FANSCAN approach instead tries to improve the segmentation also in case of heterogeneous fields with tree / crops displaces over several directions.

The CARSCAN and FANSCAN rely only on Digital Surface Model (DSM) of the study area. This is not an hard constraint considering that orthorectification engines produce orthophoto, dense cloud and also DSM. However it is possible to integrate the results of the above mentioned approaches with others based on radiometric classification.

The paper is structured as it follows. Section 2 presents the proposed approaches. Section 3 presents the results of CARSCAN and FANSCAN on two data-set in Section 4 the conclusions and future works are outlined.
\end{abstract}

\section{Methodology}

The correct tree and crop segmentation plays a key role in the domain of precision agriculture as outlined in Section 1. In this paper we outline and develop algorithms based on pure terrain based features and if possible their subsequent fusion with pure spectral approaches as in [28].

Radiometric and spectral features derived from multi/hyper-spectral images can be used by unsupervised or supervised algorithms to classify data and then select only the classes of interest to evaluate the vegetation status. Unsupervised algorithms (e.g., hierarchical clustering, ISODATA, k-means) require that the area contains objects (e.g., tree, crop, soil) that are spectrally separable. Soil response in the presence of grass could produce incorrect results considering the spectral response of bare soil with respect to one grassed over. A standard thresholding algorithm usually fails when applied to the grassed over terrain problem due to a reduction in the crop to soil area signal to noise ratio. As has already been mentioned, the presence of grass on the ground therefore strongly influences the accuracy of classification. Supervised algorithms, if properly trained are able to capture grassed soil, bare soil and tree/canopy but a common problem is the definition of a precise training set that will not underfit the problem. This requires a photo-interpretation of the area and the typical use-case for precision agriculture are small areas (from $1-1000$ hectares). A reliable training set is usually defined by a human user that should take into account local variability including spurious areas like shadows [31].

To get around this, one can use information inherent in the data itself. In this second approach, soil and tree detection is carried out by using purely mathematical features of the height field in the DSM obtained during the orthophoto generation. The effectiveness of this technology depends strongly on the scanning technique used. We investigate this dependency in detail by using a Cartesian grid scanning method to compare to a radial scanning technique over the image coordinate space. The results are theoretically connected to the object Fourier transform and this relationship is used to develop a quality index for comparing the two types of scan.

This type of analysis provides a powerful basis for precision agriculture applications that require an accurate and precise detection of crops in order to properly support decisions based on vegetational indexes that must be evaluated only on not soil areas.

The pure radiometric approach becomes challenging when the spectral response of canopy is close to the soil response. This is indeed the case for vineyards and fruit plants where the soil can be with or without grass overlay. 


\subsection{One dimensional rasterization theory}

The DSM is the output of an orthorectification engine that processes high-resolution images (with a typical GSD in the $10-50$ centimeter range). Many land areas are covered by foliage and trees, $\tau$, which obscure the underlying terrain or soil signal $\sigma$. The overall image signal is the algebraic sum of these two quantities:

$$
y(x, z)=\tau(x, z)+\sigma(x, z)
$$

Each signal is a valuable source of information and it is useful in the context of object detection to be able to separate them efficiently and accurately. For a test image like the one in Figure 1, we develop a simple and general mathematical procedure that separates the soil and tree signals into two separate digital vector fields.

The combined terrain and foliage signal $y$ is raster scanned (see Figure 1) along a coordinate direction such as $z$. Separating out the original surface $h$ into a series of sample points in the $z$-direction obtains a set of 'unrelated' one dimensional images ready to be processed independently.

Taking an arbitrary section $z=$ const across the image in Figure 1 one can reduce the soil extraction problem into a series of one dimensional sub-problems which are in theory at least, easier and faster to process.

Therefore, at some fixed $z$ :

$$
y(x)=\tau(x)+\sigma(x)
$$

where $\tau$ and $\sigma$ are the tree and soil fields across some given $z$-coordinate respectively.

The function $y$ is never in $C^{1}$ (the set of all one-fold differentiable functions). Therefore, differential methods are not general enough without significant pre-processing and a potential loss of data. The digital nature of the data does however permit the use of efficient set filters designed to separate a slow digital derivative from a relatively fast one. We will show below that this observation can be linked to statistical integral methods for solving the general problem.

One might argue that Fourier methods are also relevant here. They can be for specific cases. However, the instability of the FFT when the signal is contaminated with any significant level of noise outweighs any potential advantage a low pass filter would have. The main reason is that any attempt to control noise through expedients like Weiner or spectral filters will tend to remove high frequency detail from the image ad-lib, rendering the quickly-varying tree or contoured terrain signals inaccurate or even omitting them completely. We will show below that the use of a direct method can recover information from the Fourier space in a non-destructive fashion.

As already hinted above a more stable method is to use statistics: trees on the ground can be defined by their scatter probability density $p(x, z)$ function. The importance of this function is in defining the nearest neighbor distance from any given point $(x, z)$. Idealizing, at some such point, the tree population probability density function maximizes locally over some differential $(x+d x, z+d z)$. The associated probability density maximum is therefore constrained over some nearest neighbor contour on the $x z$-plane:

$$
\nabla p(x, z) \cdot d(x, z)=0
$$

The nearest neighbor (generally non-differentiable) probability contour serves to define a correlation distance or integral of a tree or other object class $\tau$ to its nearest neighbors. Every point on the nearest neighbor contour will tend to satisfy a maximum of this correlation integral:

$$
0=d\left[\iint \tau\left(x^{\prime}, z^{\prime}\right) \tau\left(x^{\prime}+x, z^{\prime}+z\right) d x^{\prime} d z^{\prime}\right]
$$

\footnotetext{
In the one dimensional language of Figure 3 this equation simplifies to:
} 


$$
0=\int \tau\left(x^{\prime}\right) \frac{d \tau}{d x}\left(x^{\prime}+x\right) d x^{\prime}
$$

over the object separation $w$. In other words, when this integral is at a stationary maximum, it corresponds to a local probability maximum in one dimension which dictates the local distance $w$ to a nearest neighbor for the object class $\tau$. The local spatial frequency of the object class $\tau$ at the point $x$ is:

$$
\omega_{x}=\frac{2 \pi}{w}
$$

and corresponds to the Fourier or correlation frequency of the object class $\tau$ embedded into the signal $y$. The frequency distribution of object classes on the ground gives rise to a curious relative symmetry: when the solution of equation 3 is a correlation minimum, from equation 2, the cross-correlation function of the soil will be a maximum instead:

$$
\int y\left(x^{\prime}\right) \tau\left(x^{\prime}+x\right) d x^{\prime}=\int \sigma\left(x^{\prime}\right) \tau\left(x^{\prime}+x\right) d x^{\prime}
$$

At such points $y$ is a local minimum since there is no object field, by replacing $\tau\left(x^{\prime}+x\right)$ with a normalised window $k$ of integration width $w$ :

$$
\begin{gathered}
\sigma(x, w)=y_{\min }<c \int y\left(x^{\prime}\right) k\left(x^{\prime}+x\right) d x^{\prime}= \\
\int y\left(x^{\prime}\right) \tau\left(x^{\prime}+x\right) d x^{\prime}
\end{gathered}
$$

for some constant $c$. If the integration window width is made equal to the correlation distance less the object width $b$ in the field at $x$ then the inequality is removed on the left and we have:

$$
\sigma(x, w-b)=\inf \int y\left(x^{\prime}\right) k\left(x^{\prime}+x\right) d x^{\prime}=(w-b) \min y(x)
$$

for any point $x$ that is inside the window of integration but outside the object $\tau(x)$. Applying a spline operator $S$ to the set of all points

$$
\{(x, \min \sigma(x))\}
$$

smooths the soil field data to a resolution of $\omega_{x}$ :

$$
\sigma_{S}(x)=(w-b)^{-1} S\left[\left\{\inf \int y\left(x^{\prime}\right) k\left(x^{\prime}+x\right) d x^{\prime}\right\}\right](x)
$$

where inf represents the greatest lower bound. From equation 2

$$
\tau(x)=y(x)-\sigma_{S}(x)
$$

In practice the dimension $b$ of a local object is not essential knowledge if one manipulates equation 7 into:

$$
\min y(x)=\lim _{b \rightarrow w}(w-b)^{-1} \inf \int y\left(x^{\prime}\right) k\left(x^{\prime}+x\right) d x^{\prime}=\sigma(x)
$$

where the integral is taken over the range $(x, x+n w)$ where $n \geq 2$ and $x \in\left[0, x_{\max }-n w\right]$.

The resulting set of points are solutions to equation 5 and are exactly where the correlation integral 4 of the $\tau$ object class minimises. In what follows we generalise these results over the plane in two different implementations of varying geometric complexity. The first is a Cartesian grid rasterization of the terrain and the second is its immediate generalisation or radial grid rasterization of the DSM model. The second, we will see, does indeed recover information from the original image at greater frequencies on the Fourier plane. 


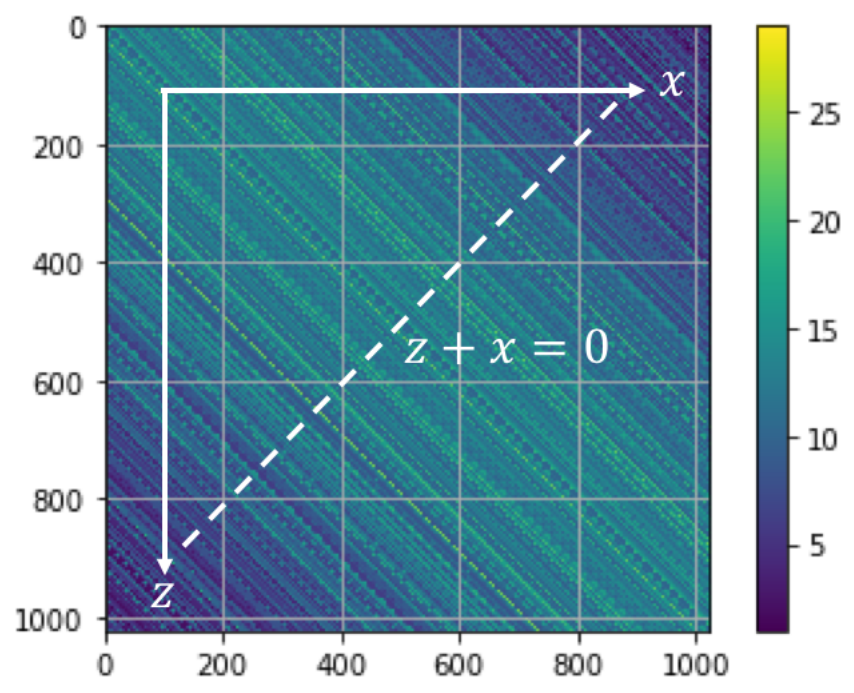

Figure 1. Gaussian test image generated artificially with a rapidly varying stochastic object field over the $z-x$ pixel plane. The $y$ height field is in arbitrary test units.

\subsection{CARSCAN: Cartesian soil field extraction}

To demonstrate the operation of these mathematical results over the DSM plane, we generate a stochastic object set (a tree field) over a Gaussian hill profile as shown in figure 1 and extract the soil and object surfaces from it. The test field image is $1024 \times 1024$ and has a rapidly varying tree or object field over the gaussian hill soil profile varying along the 45 degree $z+x=0$ diagonal (see figure 1 ).

Repeatedly extracting a sections of the field along the $z$ axis generates an array of $y_{z}(x)$ vectors along $x$. Each vector in this array can be operated on with Equation 13 to develop the soil profile at some value of $z$ as a function of $x$. Used in this way on the the entire profile array, equation 13 will generate a surface soil field at some integration window width $w$ (see equation 13). Here, instead of applying the spline operator $S$ to the one dimensional Equation 9, it is faster and more expedient from a computational point of view to apply a grid interpolation operator $G$ (written in c++ and accessed via Python's Numpy framework for example) to the soil surface data, $\sigma(x, z)$. Algorithm 1 codifies this methodology.

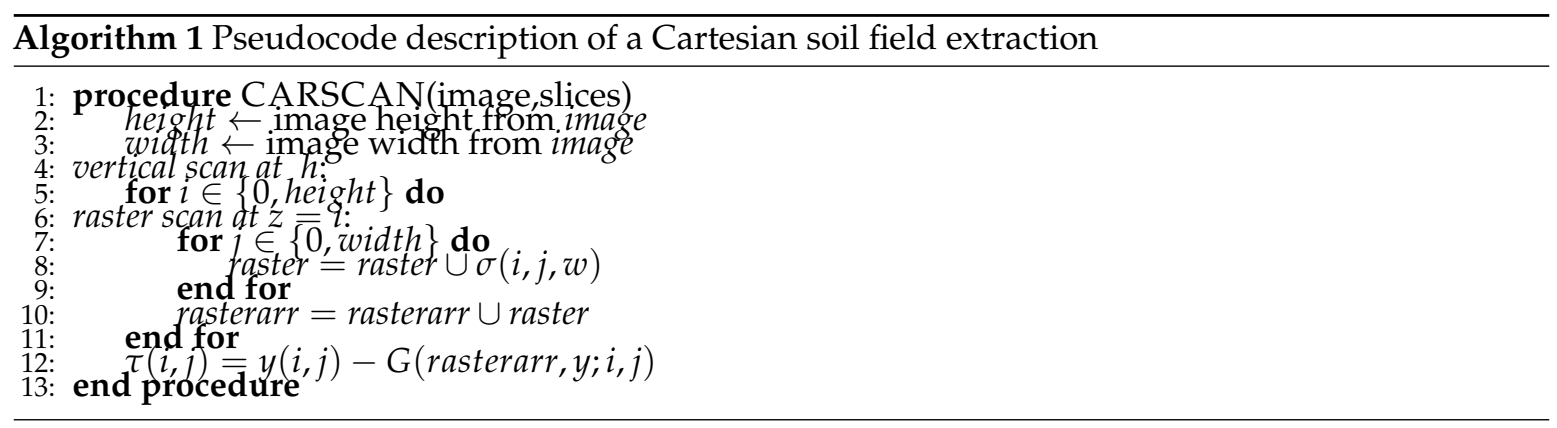

The $\sigma(x, z)$ field that results from algorithm 1 is shown in figure 2. This functional representation of the soil signal is then used to extract the object field variation over the terrain using Equation 1 directly, resulting in the object field $\tau(x, z)$ which is shown in Figure 3.

Due to the integral nature of the filter (equation 13), algorithm 1 is quite noise resistant. It is also easy to set for a variety of surfaces: for example, $w$ can be set manually or automatically to some fixed percentage of the total number of points. It is usually a good idea to set $w$ as large as the image size will permit.

Defining the characteristic function of the $\tau$ signal: 


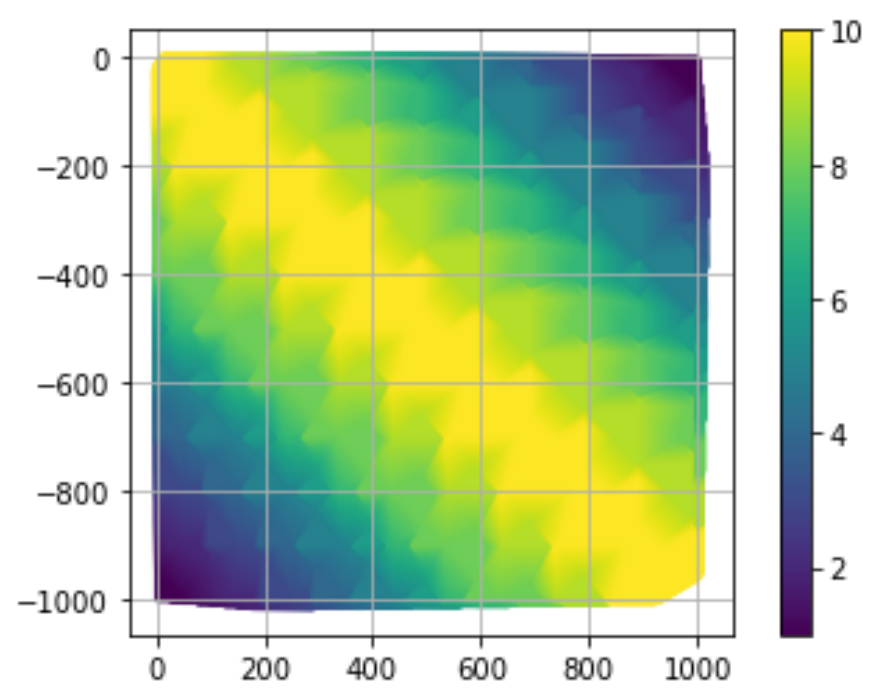

Figure 2. Soil field $\sigma(x, z)$ extraction from the original Gaussian test field image.

$$
\tau_{\chi}(x, z)= \begin{cases}0, & \tau(x, z) \leq\langle\tau(x, z)\rangle \\ 1, & \tau(x, z)>\langle\tau(x, z)\rangle\end{cases}
$$

allows a quick graphical appreciation of the object detection/classification area in the DSM model. This is calculated in Figure 4 when the threshold level is set to the mean object field height.

\subsection{FANSCAN: Moving radial soil field extraction}

The integration method when generalised over many rasters line provides a convenient recipe for separating the aerial image into object and soil fields as was shown in the Carscan algorithm above. This Cartesian strategy can infact be envisaged along any direction in the image to yield information particular to that orientation. The advantage of such rasterised vectoring (or radial scanning) of the image is that it produces more information about the image frequencies in an off axis direction and is therefore akin to a high resolution Fourier sampling of the ground object frequencies $\omega_{L}$ along some line $L$. The essential difference is that this is a direct and hence more stable methodology for sampling, with the advantage that the numerical errors commonly associated with passages into and out of transform spaces can be avoided while collecting information on those frequencies. An algorithm designed around this principle would in theory be capable of obtaining the most complete directional frequency scan of an image in direct space.

One method of achieving this is to make the series of direct horizontal rasters across the image in the CARSCAN algorithm act as seeds for such a strategy. A given raster at $(x=0, z)$ can be rotated along any direction $\mathbf{v}$ in the image and rasterized to develop a one dimensional picture of the object distribution along that line. Equation 14 would then develop the object and soil extractions for the raster as planned earlier but in the direction $\mathbf{v}$. Fanning the original raster $(x=0, z)$ along all the possible directions $\mathbf{v}$ forms a basis for the FANSCAN algorithm presented here (see algorithm 2 and figure 5).

FANSCAN delivers, therefore, the entire image surface as a series of raw data points classified along their raster directions through the fan or direction vector $\mathbf{v}$ (we take this symbol to mean both a direction or discretization set of vectors as will be apparent from the context). Equation 13 applied along any of these directions extracts the soil component of the raster and can be used to develop a directionally sensitive picture of the soil structure at any point in the image. The data that contains this information is a three dimensional point cloud which can be interpolated to fit the original point cloud of the raw image to extract a directionally rich soil field $\sigma_{\mathbf{v}}(x, z)$. 


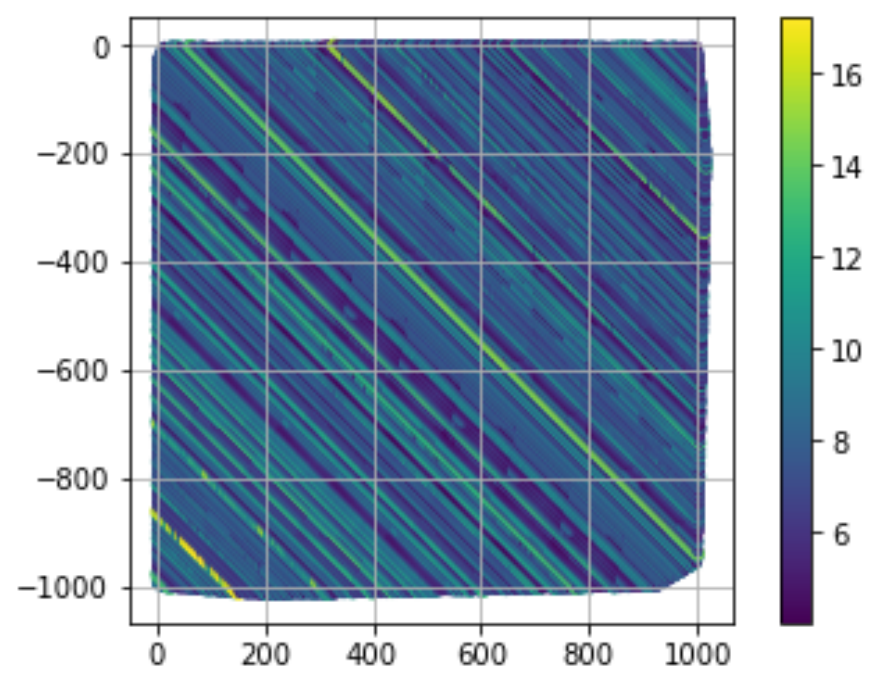

Figure 3. Object field $\tau(x, z)$ derived from equation 3. Notice that the correlation frequency for these objects is constant everywhere along $z+x=0$.

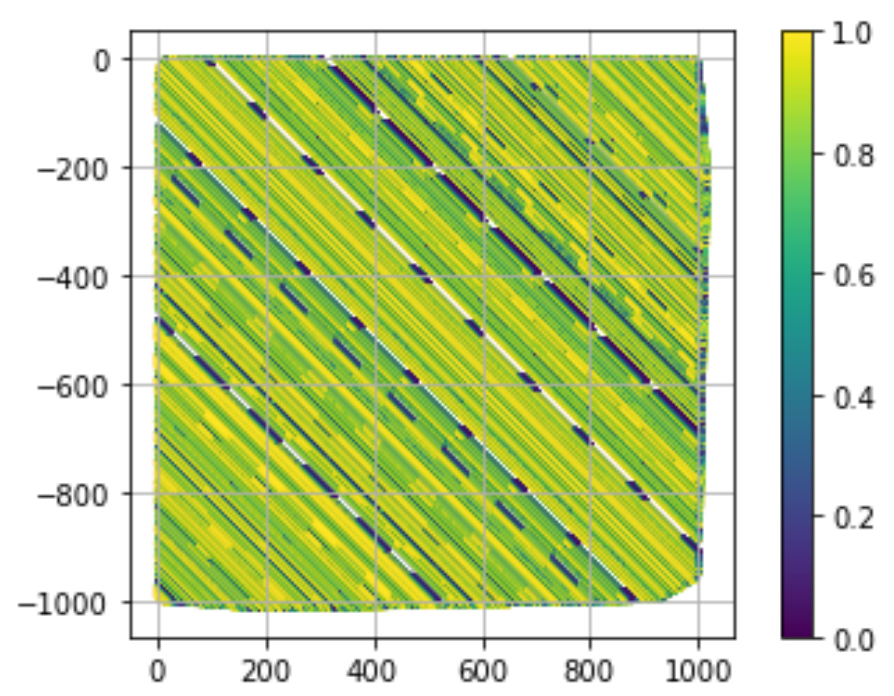

Figure 4. The results of applying equation thresholding to $\tau(x, z)$ in figure 3 at the mean object field height is the membership function $\tau_{\chi}(x, z)$. Notice that the correlation frequency for these objects is constant everywhere along $z+x=0$.

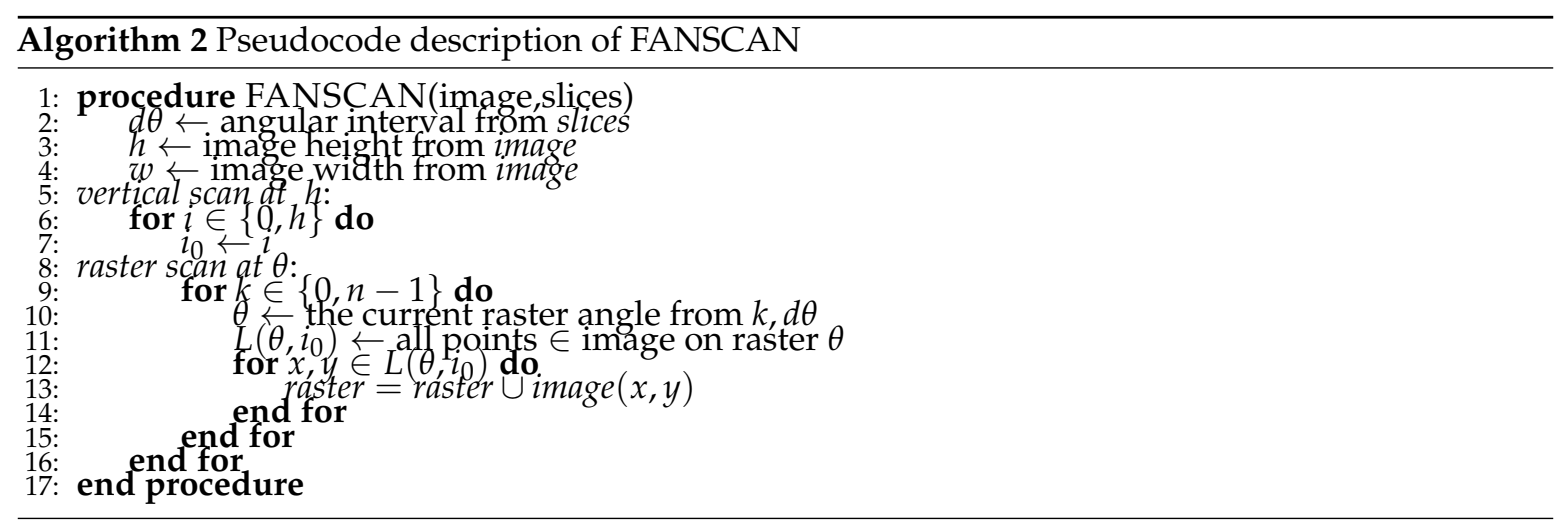




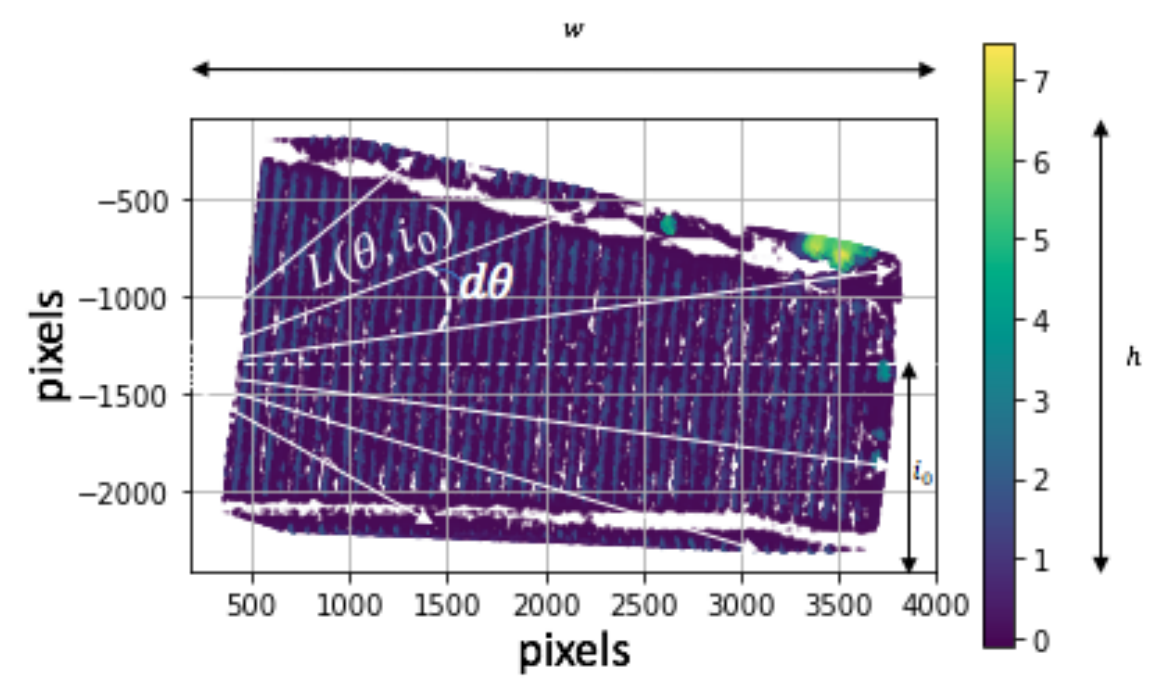

Figure 5. Geometry of the FANSCAN algorithm (see algorithm 2). The white arrows are the raster vectors $\mathbf{v}$ across an extracted object field DSM. The dotted horizontal line is the current vertical scan position. Negative pixels on the $z$ axis are an artefact of matrix to image reflection. The vertical colorbar is in metres.

Once the DSM source $\sigma_{\mathbf{v}}(x, z)$ has been extracted from the FANSCAN algorithm in this way, the original image and it can be subtracted over the plane to extract the three dimensional point cloud that is in fact a high resolution object field $\tau_{\mathbf{v}}(x, z)$ of the image in direct space.

In the context of this paper, we monitor the efficiency of the the algorithm as a function of the discretization of the vector sets $\mathbf{v}$ to derive a relative extraction metric for the algorithm. Since the theoretical benefit of using a radial scan in this manner is to provide more information on directional object frequencies, such a metric can be naturally specified in terms of Fourier space frequencies already introduced in equation 6 for the direction $x$.

Defining the Fourier space efficiency (or frequency reach) of a FANSCAN extraction $\eta$ over some set of discrete vectors $\mathbf{v}$ as:

$$
\eta\left(\mathbf{v}, \mathbf{v}_{\infty}\right)=1-\frac{\left\|\mathrm{FFT}_{\infty}-\mathrm{FFT}_{\mathbf{v}}\right\|_{2}}{\left\|\mathrm{FFT}_{\infty}\right\|_{2}}
$$

where $\mathrm{FFT}_{\infty}$ is the fast fourier transform of the original image and $\mathrm{FFT}_{\mathbf{v}}$ is the computed fast Fourier transform of $\sigma_{\mathbf{v}}(x, z)+\tau_{\mathbf{v}}(x, z)$, provides one such method for measuring the performance of the extraction algorithm.

Equation 15 is a theoretical construct that is difficult to calculate since algorithm 2 extracts the object field by computing the soil surface first. That is to say, the efficiency of the operation can only be measured if the true soil surface were known, which it is not. However, there is a way around that problem if we rewrite equation 15 as a sequence for the extracted object field only:

$$
\eta\left(\mathbf{v}_{i}, \mathbf{v}_{\mathbf{i}+\mathbf{1}}\right)=\frac{\left\|\mathrm{FFT}_{\mathbf{v}_{\mathbf{i}}}\right\|_{2}}{\left\|\mathrm{FFT}_{\mathbf{v}_{\mathbf{i}+\mathbf{1}}}\right\|_{2}}
$$

If the sequence of images generated by the FANSCAN algorithm is convergent in the space of images (easy to prove) then:

$$
\lim _{i \rightarrow \infty} \eta\left(\mathbf{v}_{i}, \mathbf{v}_{\mathbf{i}+\mathbf{1}}\right)=\mathbf{1}
$$


This relationship is dependent on the asymptotic convergence of the sequence of Fourier transforms and is related to the convergence efficiency $\eta\left(\mathbf{v}_{0}, \mathbf{v}_{\infty}\right)$ at the endpoints by:

$$
\eta\left(\mathbf{v}_{0}, \mathbf{v}_{\infty}\right)=\prod_{i=0}^{\infty} \eta\left(\mathbf{v}_{i}, \mathbf{v}_{\mathbf{i}+\mathbf{1}}\right)
$$

A similar line of reasoning shows that the following general condition, where $\mathbf{v}_{\mathbf{0}}$ is the simple CARSCAN algorithm across the image, will be observed:

$$
\lim _{N \mathbf{v} \rightarrow \infty} \frac{\partial \eta\left(\mathbf{v}_{0}, \mathbf{v}\right)}{\partial N \mathbf{v}}=\mathbf{0}
$$

where the algorithm has converged and by $N \mathbf{v}$ we mean the resolution or number of vectors in set $\mathbf{v}$. It is therefore clear that $\partial \eta\left(\mathbf{v}_{0}, \mathbf{v}\right)$ measures the quality of the processing operation between the initial (CARSCAN) image result and the FANSCAN results when $i>0$.

\section{Results and Discussion}

\subsection{Data-set}

The study areas are located on a hilly farmland area. The acquisition campaigns were performed with an AscTec Pelican equipped with the Sequoia multi-spectral camera. Figure 6 shows the study areas and the related DSM. The final ortho products have a final Ground Sampling Distance (GSD) of 4 centimeters with 0.5 meters of horizontal accuracy.In both data-sets we planned to have a lateral and longitudinal overlap above the $70 \%$.

The quality of acquired data reflects on both orthophoto and DSM. Quality is mainly influenced by the attitude of vehicle during the acquisition, height above the ground. This last aspect plays a key role especially in hilly areas. If the mission was planned with a constant height each single image will have a different GSD especially in areas with high slopes. We tried to set-up the acquisition by using a constant height above the ground even if this required an a priori knowledge of the DEM of area.

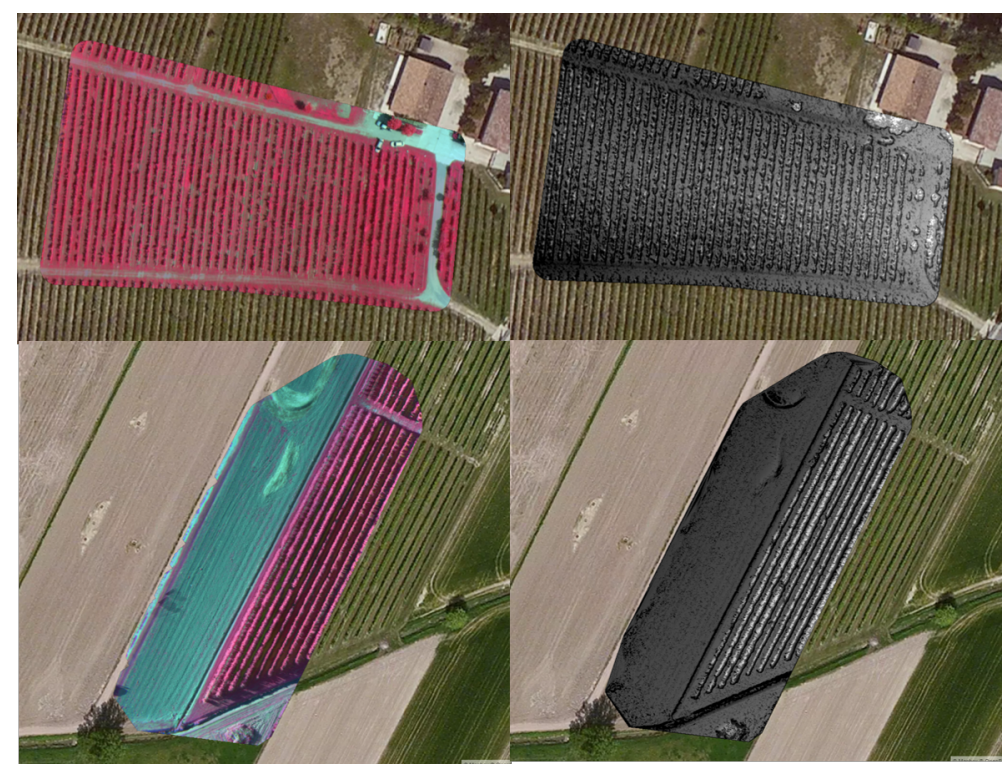

Figure 6. Left. Study area 1 Top: derived orthophoto of vineyard area with false color (left) and derived DSM (right; black represents low height). Study area 2 Top: derived orthophoto of plant fruit area with false color (left) and derived DSM (right; black represents low height). 
Study area 1 represents a hilly area of vineyards where several rows of trees are present also with different displacement in the top area. Trees have an average height above the ground of 2 meters with a small canopy at the top $(0.7 \mathrm{~m})$.

Study area 2 represents an area covered by fruit plants with a small and constant slope over the area. Trees have an average height above the ground of 2.5 metres with a large canopy at the top (up to $3 \mathrm{~m}$ ).

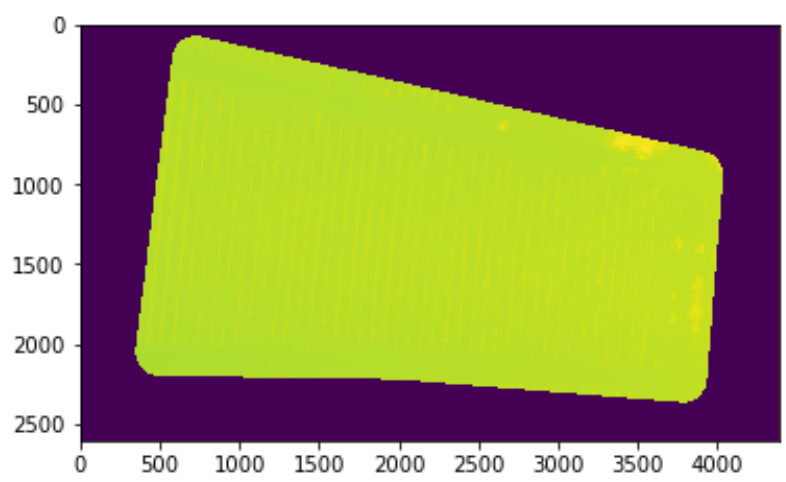

Figure 7. The first study area data set for testing the scanning algorithms; DSM Field at $2604 \times 4381$ pixels. The object field plantation ridges are barely visible to eye without segmentation. Equation 14 can extract them efficiently nonetheless.

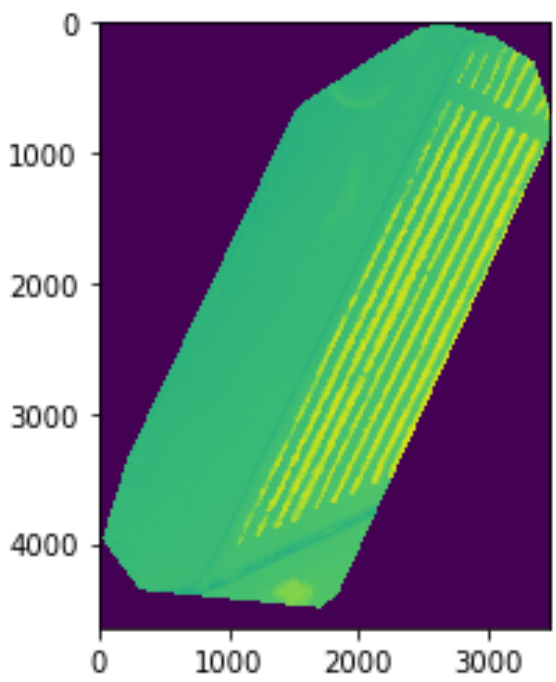

Figure 8. The second study area data set for testing the scanning algorithms; DSM at $4645 \times 3465$ pixels. This is a simple terrain map whose orientation exposes a flaw in the FANSCAN algorithm design.

\subsection{FANSCAN vs CARSCAN}

Using the same image DSM image as in Figure 6 and applying algorithm 2 obtains the interpolated soil surface $\sigma_{\mathbf{v}}(x, z)$ as shown in figure 11 . The extracted object field $\tau_{\mathbf{v}}(x, z)$ is given in figure 12 . The extraction metric for this image can be seen in figure 14 .

To test and illustrate the method further we include a second DSM data-set seen in figure 8 .

Running FANSCAN on this data shows the theoretical consistency of the method and at the same time an apparent weakness in its design.

When a raster vector $\mathbf{v}$ falls directly upon a row of trees, the soil extraction as developed in equation 13 will fail. This aspect is nicely illustrated in figure 17 for the second dataset in figure 8 where part of the object field gets extracted out with the soil field at around $N \mathbf{v}=100$ fans. 


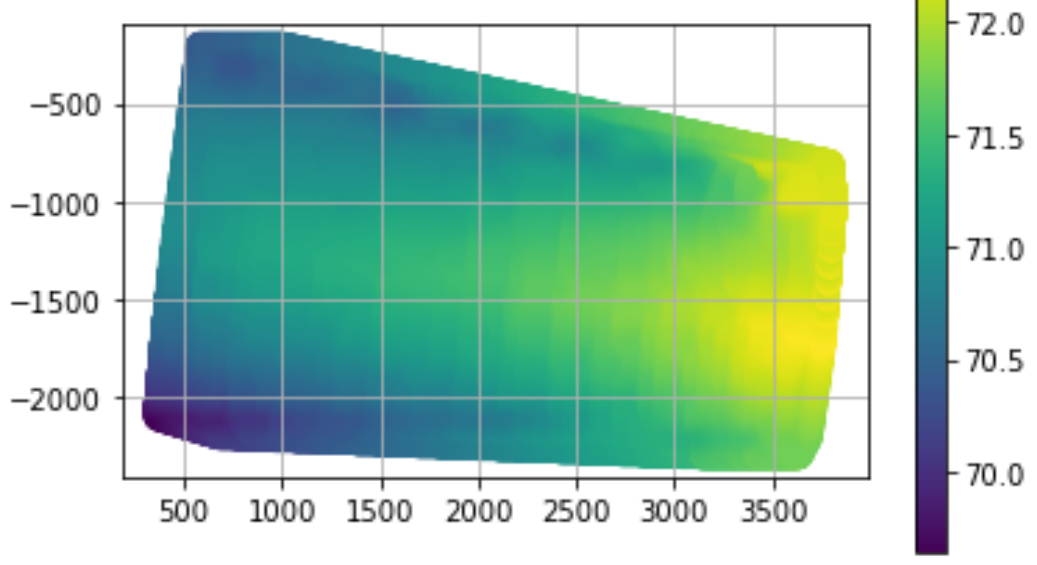

Figure 9. The result of the FANSCAN soil extraction applied to figure 7 at $N \mathbf{v}=1$ fan rasters per horizontal seed point. This corresponds to the $\mathbf{v}_{0}$ CARSCAN algorithm in the example above. The colour scale is in meters and negative pixel numbers are an artifact of the image to matrix conversion.

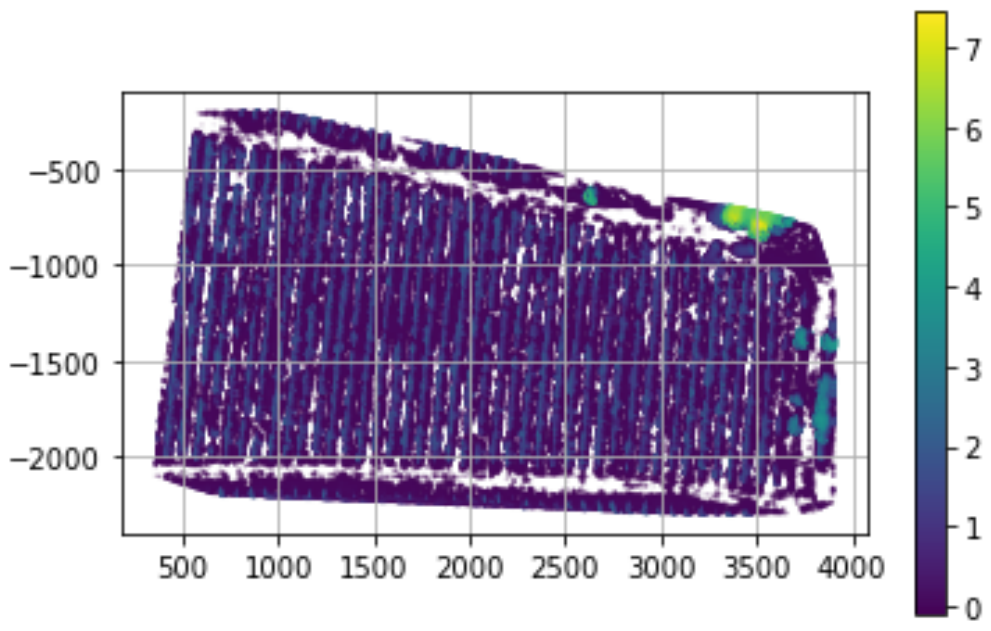

Figure 10. The result of the FANSCAN object extraction applied to figure 7 at $N \mathbf{v}=1$ fan rasters per horizontal seed point. This corresponds to the $\mathbf{v}_{0}$ CARSCAN algorithm in the example above. The colour scale is in meters and negative pixel numbers are an artifact of the image to matrix conversion.

There are several solutions to this problem and all of them involve avoiding an encounter with such a situation in the first place. The first possibility is to limit the maximum resolution (discretization of the fanscan) manually. The second is to randomize both the horizontal seeding and the FANSCAN rasterization. A combination of both of these measures can produce good results for the simple test images as studied here but will fail in places for complex object field extractions.

The most costly, but a guaranteed solution, is to search successive soil field approximations for competing minima and to reject any outliers from the soil field sequence. There are however considerable difficulties in achieving this: the main one being that the physical number of points in each extracted image is different and therefore extensive use of back interpolation needs to be made to coregister the entire sequence being considered for correction. That can require lots of memory (gigabytes) for even the most modest of images. 


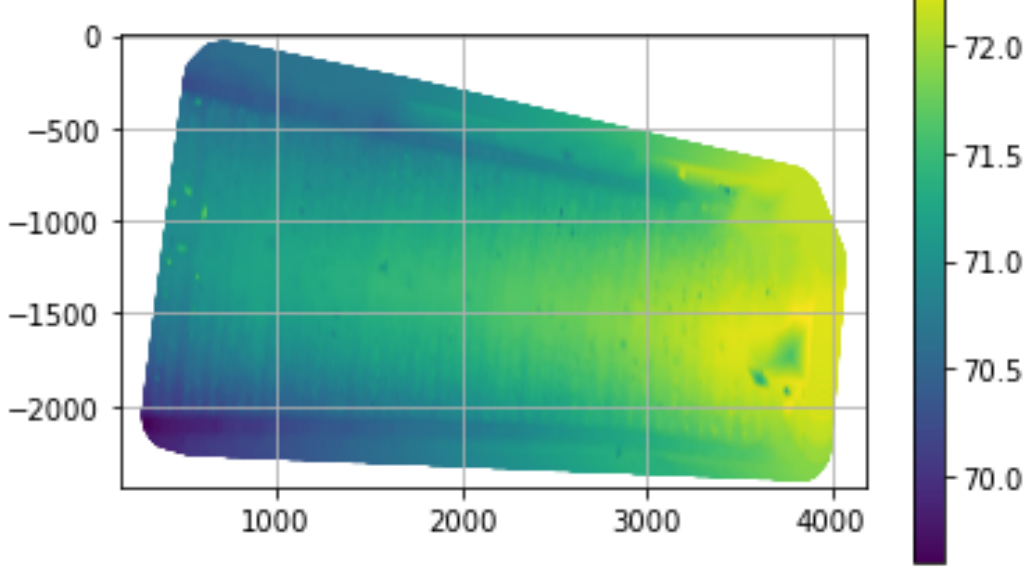

Figure 11. The result of the FANSCAN soil extraction applied to figure 7 at $N \mathbf{v}=100$ fan rasters per horizontal seed point. The colour scale is in meters and negative pixel numbers are an artifact of the image to matrix conversion.

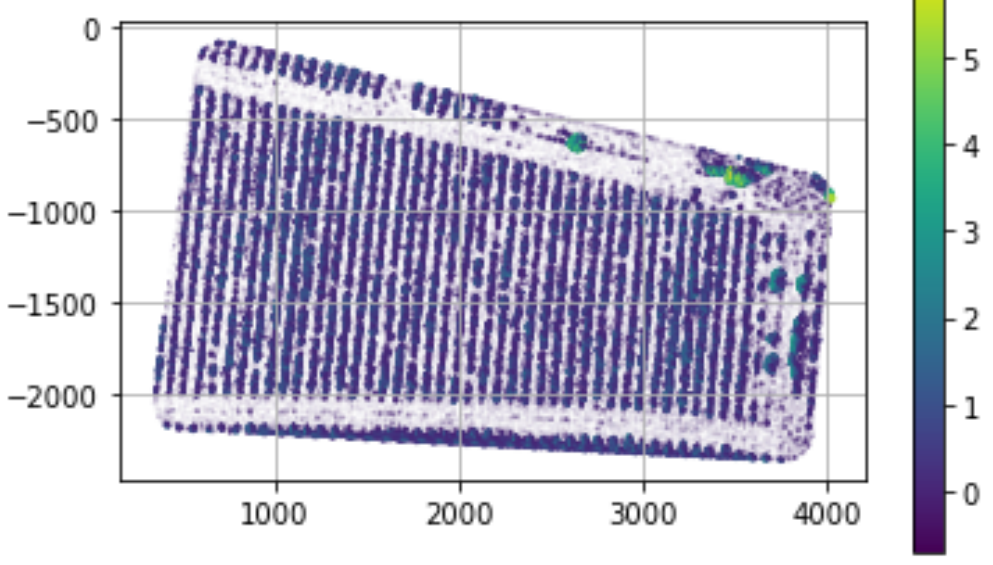

Figure 12. The result of the FANSCAN object extraction applied to figure 7 at $N \mathbf{v}=100$ fan rasters per horizontal seed point. The colour scale is in meters and negative pixel numbers are an artifact of the image to matrix conversion.

While a fully automated solution can take time, in essence all that is actually required is one artifact free image from the sequence so that artifacts in the sequence can be automatically recognized and then removed. Following the discussion above, a good candidate for that image is the very first (CARSCAN) iteration : $\mathbf{v}_{0}$. The logical matrix operation:

$$
\sigma_{\mathbf{v}_{i+1}}^{\prime}=\left(\sigma_{\mathbf{v}_{0}} \geq \sigma_{\mathbf{v}_{i+1}}\right) \sigma_{\mathbf{v}_{i+1}}+\left(\sigma_{\mathbf{v}_{0}}<\sigma_{\mathbf{v}_{i+1}}\right) \sigma_{\mathbf{v}_{0}}
$$

will quickly post process and correct the artifacts from the soil field. Figure 21 shows this correction process applied to get back the corrected soil field for the FANSCAN at $N \mathbf{v}=100$. The multiplicity of rasters across the object field make it highly unlikely that the object field is adversely affected by this phenomenon, so no correction need be applied. However, should one be necessary, it is easily generated along with the soil field correction itself as shown in figure 22. 


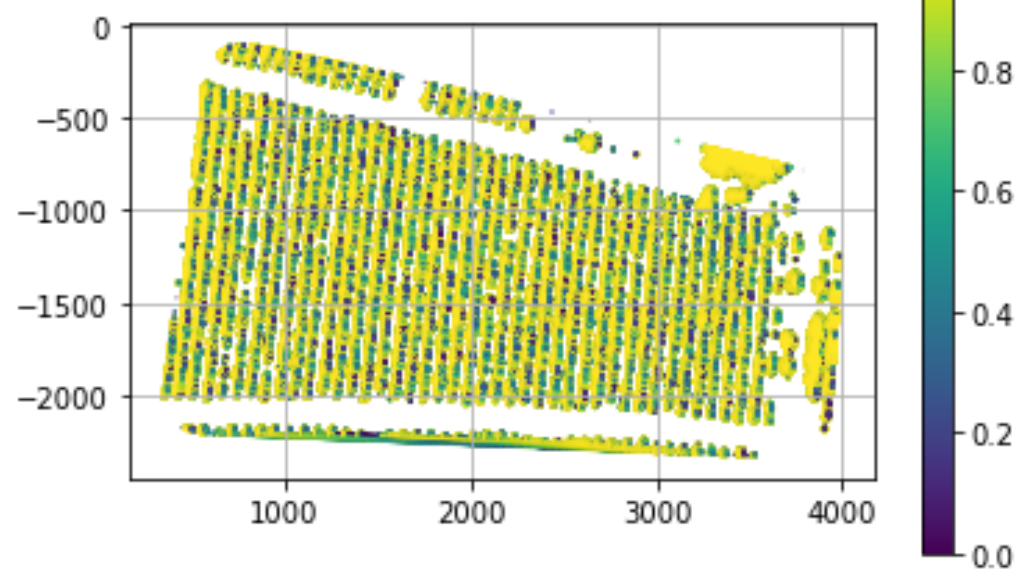

Figure 13. The FANSCAN object characteristic applied to figure 7 at $N \mathbf{v}=100$ fan rasters per horizontal seed point. The colour scale is in meters and negative pixel numbers are an artifact of the image to matrix conversion.

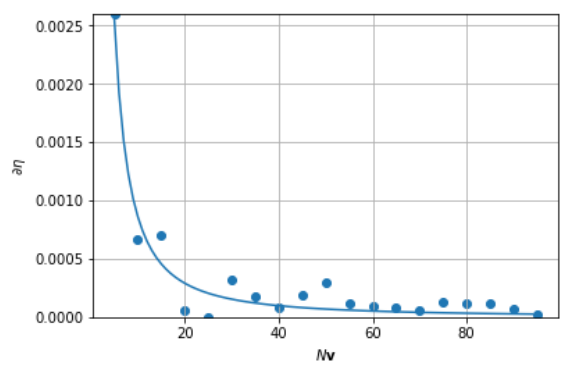

Figure 14. Equation 19 in practice for the DSM data of figure 7: the closer the points are to the abscissa, the better the quality (convergence) of the image. The solid blue line is a power law nonlinear regression for the measured data and shows the likely value of the quality metric as a continuous function of $N \mathbf{v}$.

The theoretical basis of all these considerations is demonstrated by Equation 19 in the form of plots of $\partial \eta$ shown for both data sets (see figures 14, 20). Moving backwards along the abcissa and hence reducing the raster discretization to zero (that is towards the CARSCAN rasterization) shows an accompanying depreciation in the Fourier space reach of the algorithm. In both cases the overall accuracy evaluated over a ground truth as described in [28] is above the $95 \%$.

\section{Conclusions}

In this paper we have presented two algorithms to segment crops and/or tree objects over soil by using high-resolution images starting from Digital Surface Models that are usually available when the data have been acquired by using unmanned platforms.

The approach is based on a two dimensional data slicing or reduction technique. Each slice is separately processed as a one dimensional time series to derive the terrain and tree structures separately, here interpreted as object probability densities. The results demonstrate that the method potentially enables the correct segmentation of soil and can thus offer insights into the geometric distribution of surface objects upon it.

A more sophisticated variant of this idea is the FANSCAN algorithm introduced above (see figure 5 and algorithm 2). It uses vector or radial raster scanning across the image to increase the frequency resolution of the scanned data. The results are a generated sequence of images that converge onto the 


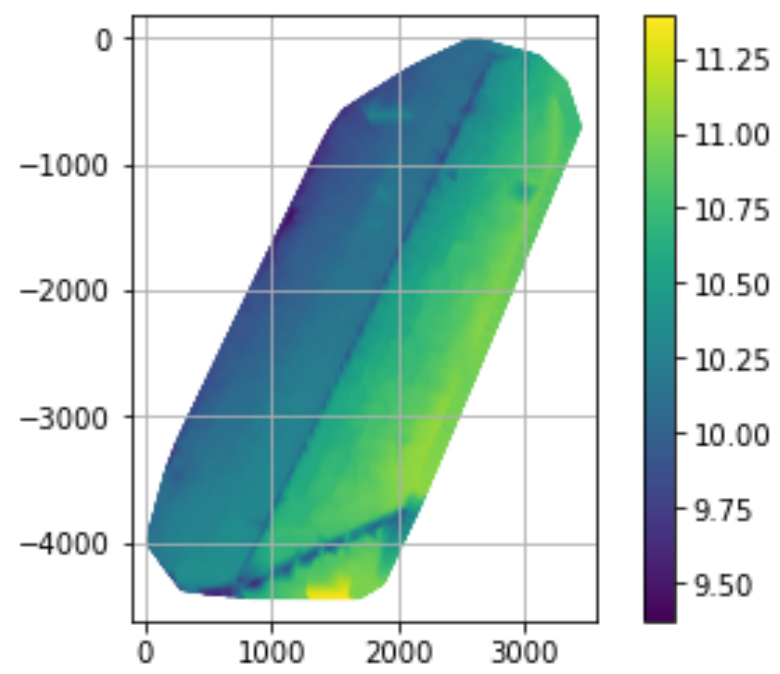

Figure 15. The result of the FANSCAN soil extraction applied to figure 8 at $N \mathbf{v}=1$ fan rasters per horizontal seed point. This corresponds to the $\mathbf{v}_{0}$ CARSCAN algorithm in the example above. The colour scale is in meters and negative pixel numbers are an artifact of the image to matrix conversion.

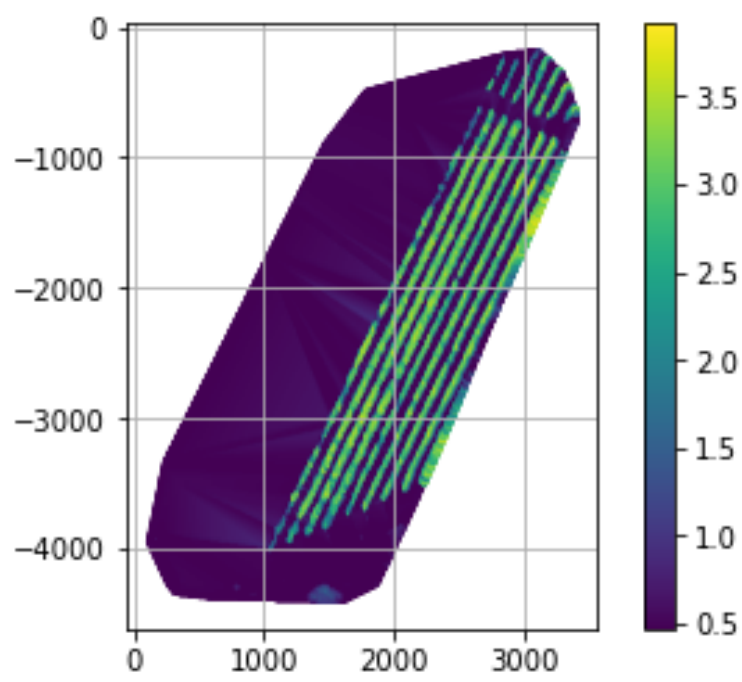

Figure 16. The result of the FANSCAN object extraction applied to figure 8 at $N \mathbf{v}=1$ fan rasters per horizontal seed point. This corresponds to the $\mathbf{v}_{0}$ CARSCAN algorithm in the example above. The colour scale is in meters and negative pixel numbers are an artifact of the image to matrix conversion.

original image. The frequency performance of the derived object field sequence was measured using a Fourier efficiency metric which vanishes at infinite time.

Due to real world considerations it would be prudent to ally the quality metric with a measure of the number of processor cycles at time $t$ to define an overall functional of performance. The unique limit point of the image sequence in direct and Fourier spaces means that such a functional would be a global optimizer for the algorithm.

An apparent drawback of the FANSCAN algorithm is that it will run into trouble when it encounters a coincident object field line (such as an avenue of trees) as has been seen in figure 17. If a raster line lies on top of one of these arrays then the soil extractor will suddenly reduce its efficiency and real objects will tend to creep into the soil field. A costly, but accurate method for dealing with these situations is to post process the image against a lower resolution image soil field construction where 


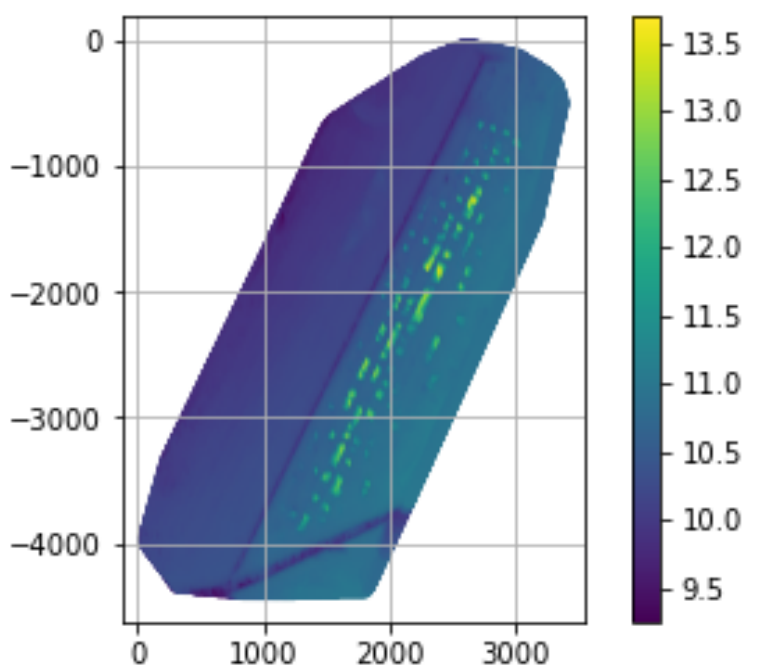

Figure 17. The result of the FANSCAN soil extraction applied to figure 8 at $N \mathbf{v}=100$ fan rasters per horizontal seed point. Note how certain parts of the object field have been included in the soil extraction. The colour scale is in meters and negative pixel numbers are an artifact of the image to matrix conversion.

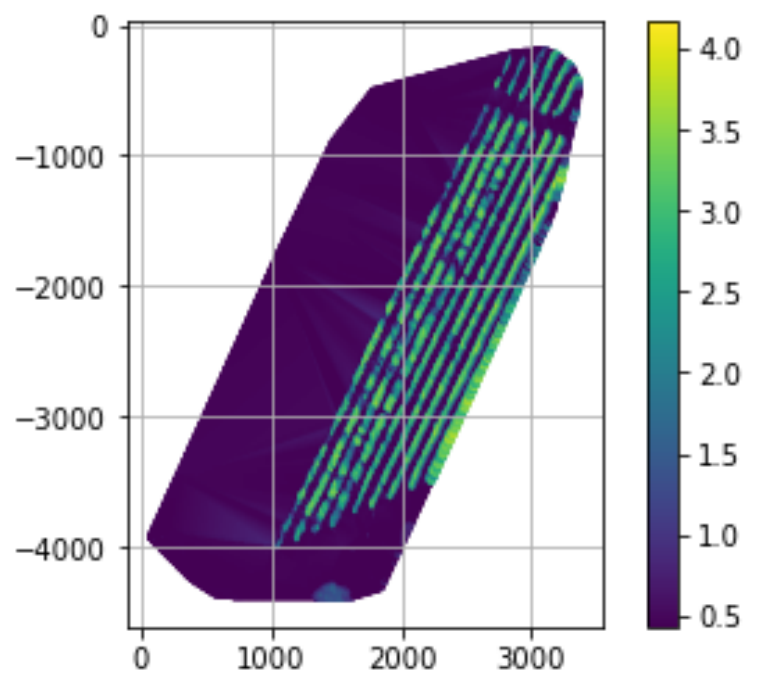

Figure 18. The result of the FANSCAN object extraction applied to figure 8 at $N \mathbf{v}=100$ fan rasters per horizontal seed point. The colour scale is in meters and negative pixel numbers are an artifact of the image to matrix conversion.

raster discretization avoids this situation. Cross elimination of coincident maxima then removes the artifacts and both the object and soil fields can thus be corrected at higher resolution scans. Equation 20 is an example of one such measure. Of course, a fairly convergent low resolution FANSCAN lowers the probability of this occurring. An added bonus is that the same strategy lowers the runtime for the algorithm. For these reasons, a high resolution FANSCAN is not in general recommended.

For upcoming research we will perform more tests also evaluating a pure random approach in terms of radial direction and radial ray's start that tries to mix the advantages of CARSCAN and FANSCAN.

Acknowledgments: The authors would like to thank Carlo Alberto Bozzi of EVE S.r.l. for his valuable support during the preparation of this article. 


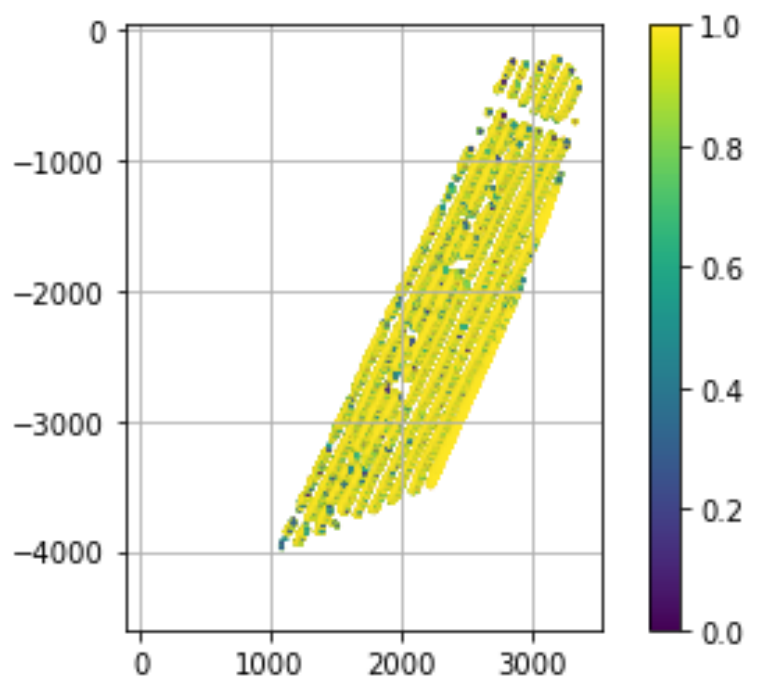

Figure 19. The FANSCAN object characteristic applied to figure 8 at $N \mathbf{v}=100$ fan rasters per horizontal seed point. The colour scale is in meters and negative pixel numbers are an artifact of the image to matrix conversion.

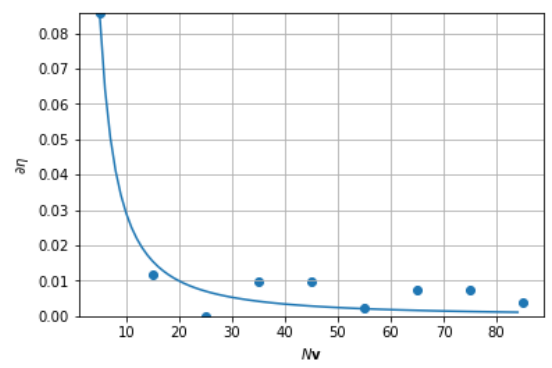

Figure 20. Equation 19 in practice for the DSM data of figure 8: the closer the points are to the abcissa, the better the quality (convergence) of the image. The solid blue line is a power law nonlinear regression for the measured data and shows the likely value of the quality metric as a continuous function of $N \mathbf{v}$.

Author Contributions: Professors Frontoni and Zingaretti are responsible for carrying out research on the various segmentation approaches referred to in this article. Prof. Adriano Mancini and Dr Jack Dyson have worked on the rasterization analysis and extraction techniques for the $\sigma$ and $\tau$ digital vector fields used in this work.

1. Colomina, I.; Molina, P. Unmanned aerial systems for photogrammetry and remote sensing: A review. \{ISPRS\} Journal of Photogrammetry and Remote Sensing 2014, 92, 79 - 97.

2. Enciso, J.; Maeda, M.; Landivar, J.; Avila, C.; Jung, J.; Chang, A. Unmanned aerial system (UAS) for precision agriculture and management decisions. 2016.

3. Candiago, S.; Remondino, F.; De Giglio, M.; Dubbini, M.; Gattelli, M. Evaluating Multispectral Images and Vegetation Indices for Precision Farming Applications from UAV Images. Remote Sensing 2015, 7, 4026.

4. Matese, A.; Toscano, P.; Di Gennaro, S.F.; Genesio, L.; Vaccari, F.P.; Primicerio, J.; Belli, C.; Zaldei, A.; Bianconi, R.; Gioli, B. Intercomparison of UAV, Aircraft and Satellite Remote Sensing Platforms for Precision Viticulture. Remote Sensing 2015, 7, 2971-2990.

5. Bernardini, A.; Frontoni, E.; Malinverni, E.; Mancini, A.; Tassetti, A.; Zingaretti, P. Pixel, object and hybrid classification comparisons. Journal of Spatial Science 2010, 55, 43-54.

6. Hedley, C. The role of precision agriculture for improved nutrient management on farms. Journal of the Science of Food and Agriculture 2015, 95, 12-19. 


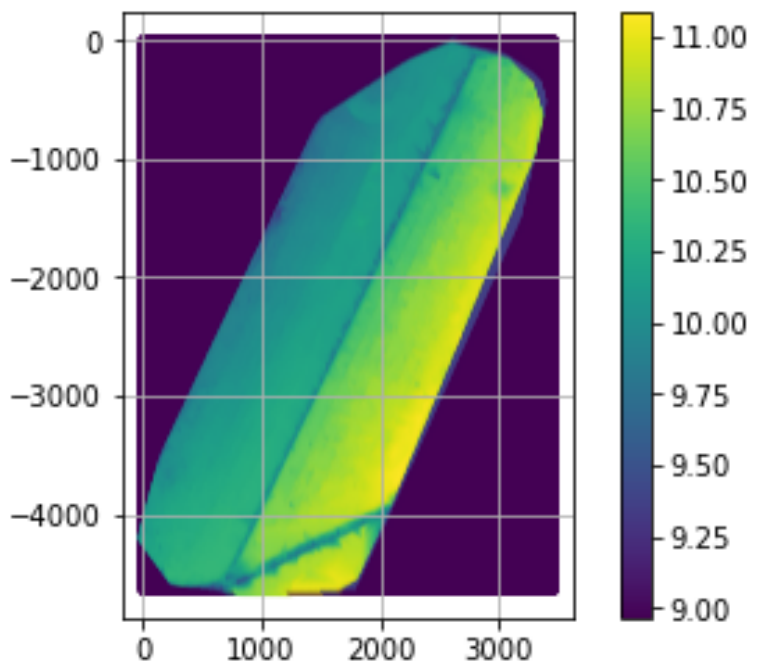

Figure 21. The result of the FANSCAN soil field correction applied to figure 17 at $N \mathbf{v}=100$ fan rasters per horizontal seed point. The correction eliminates the parts of the object field included into the image from figure 18. The colour scale is in meters and negative pixel numbers are an artifact of the image to matrix conversion.

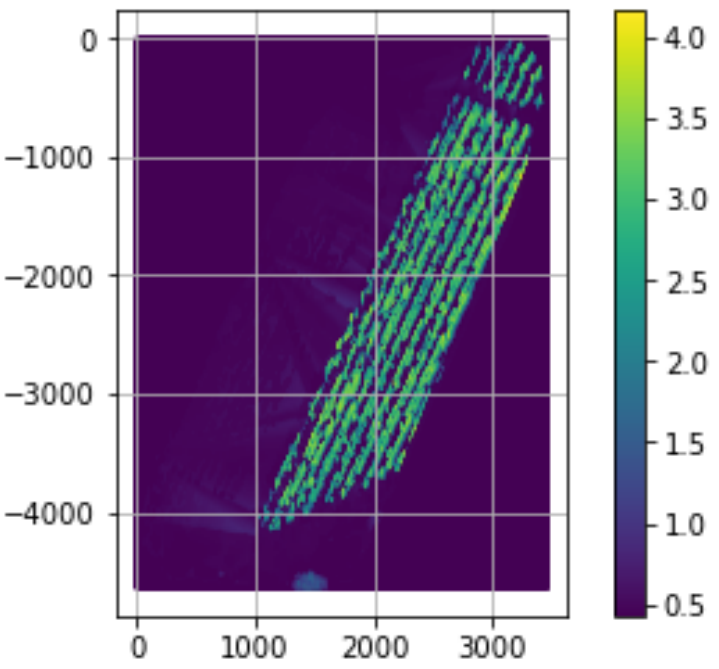

Figure 22. The result of the FANSCAN object field correction applied to figure 8 at $N \mathbf{v}=100$ fan rasters per horizontal seed point. The correction adds the parts of the object field included into the image from figure 18. The colour scale is in meters and negative pixel numbers are an artifact of the image to matrix conversion.

7. Schenatto, K.; de Souza, E.; Bazzi, C.; Gavioli, A.; Betzek, N.; Beneduzzi, H. Normalization of data for delineating management zones. Computers and Electronics in Agriculture 2017, 143, 238-248.

8. Jin, Z.; Prasad, R.; Shriver, J.; Zhuang, Q. Crop model- and satellite imagery-based recommendation tool for variable rate $\mathrm{N}$ fertilizer application for the US Corn system. Precision Agriculture 2017, 18, 779-800.

9. De Benedetto, D.; Castrignanò, A.; Rinaldi, M.; Ruggieri, S.; Santoro, F.; Figorito, B.; Gualano, S.; Diacono, M.; Tamborrino, R. An approach for delineating homogeneous zones by using multi-sensor data. Geoderma 2013, 199, 117-127. 
10. Yang, C.; Odvody, G.; Fernandez, C.; Landivar, J.; Minzenmayer, R.; Nichols, R. Evaluating unsupervised and supervised image classification methods for mapping cotton root rot. Precision Agriculture 2015, $16,201-215$.

11. Zurita-Milla, R.; Izquierdo-Verdiguier, E.; De By, R. Identifying crops in smallholder farms using time series of WorldView-2 images. 2017.

12. Varela, S.; Assefa, Y.; Vara Prasad, P.; Peralta, N.; Griffin, T.; Sharda, A.; Ferguson, A.; Ciampitti, I. Spatio-temporal evaluation of plant height in corn via unmanned aerial systems. Journal of Applied Remote Sensing 2017, 11.

13. Iqbal, F.; Lucieer, A.; Barry, K.; Wells, R. Poppy crop height and capsule volume estimation from a single UAS flight. Remote Sensing 2017, 9.

14. Erten, E.; Lopez-Sanchez, J.; Yuzugullu, O.; Hajnsek, I. Retrieval of agricultural crop height from space: A comparison of SARtechniques. Remote Sensing of Environment 2016, 187, 130-144.

15. Aasen, H.; Burkart, A.; Bolten, A.; Bareth, G. Generating 3D hyperspectral information with lightweight UAV snapshot cameras for vegetation monitoring: From camera calibration to quality assurance. ISPRS Journal of Photogrammetry and Remote Sensing 2015, 108, 245-259.

16. De Souza, C.; Lamparelli, R.; Rocha, J.; Magalhães, P. Height estimation of sugarcane using an unmanned aerial system (UAS) based on structure from motion (SfM) point clouds. International Journal of Remote Sensing 2017, 38, 2218-2230.

17. Chaves, A.; La Scalea, R.; Colturato, A.; Kawabata, C.; Furtado, E.; Branco, K. Using UAVs and digital image processing to quantify areas of soil and vegetation. Journal of Physics: Conference Series 2015, 633.

18. Yue, J.; Yang, G.; Li, C.; Li, Z.; Wang, Y.; Feng, H.; Xu, B. Estimation of winter wheat above-ground biomass using unmanned aerial vehicle-based snapshot hyperspectral sensor and crop height improved models. Remote Sensing 2017, 9.

19. Malambo, L.; Popescu, S.; Murray, S.; Putman, E.; Pugh, N.; Horne, D.; Richardson, G.; Sheridan, R.; Rooney, W.; Avant, R.; Vidrine, M.; McCutchen, B.; Baltensperger, D.; Bishop, M. Multitemporal field-based plant height estimation using $3 \mathrm{D}$ point clouds generated from small unmanned aerial systems high-resolution imagery. International Journal of Applied Earth Observation and Geoinformation 2018, 64, 31-42.

20. Torres-Sanchez, J.; Lopez-Granados, F.; Serrano, N.; Arquero, O.; Pena, J. High-throughput 3-D monitoring of agricultural-tree plantations with Unmanned Aerial Vehicle (UAV) technology. PLoS ONE 2015, 10. cited By 20.

21. Lopez-Granados, F.; Torres-Sanchez, J.; De Castro, A.I.; Serrano-Perez, A.; Mesas-Carrascosa, F.J.; Pena, J.M. Object-based early monitoring of a grass weed in a grass crop using high resolution UAV imagery. Agronomy for Sustainable Development 2016, 36.

22. Perez-Ortiz, M.; Pena, J.; Gutierrez, P.; Torres-Sanchez, J.; Hervas-Martinez, C.; Lopez-Granados, F. Selecting patterns and features for between- and within- crop-row weed mapping using UAV-imagery. Expert Systems with Applications 2016, 47, 85-94.

23. Perez-Ortiz, M.; Pena, J.; Gutierrez, P.; Torres-Sanchez, J.; Hervas-Martinez, C.; Lopez-Granados, F. A semi-supervised system for weed mapping in sunflower crops using unmanned aerial vehicles and a crop row detection method. Applied Soft Computing Journal 2015, 37, 533-544.

24. Hamuda, E.; Mc Ginley, B.; Glavin, M.; Jones, E. Automatic crop detection under field conditions using the HSV colour space and morphological operations. Computers and Electronics in Agriculture 2017, 133, 97-107.

25. Kiani, S.; Jafari, A. Crop Detection and Positioning in the Field Using Discriminant Analysis and Neural Networks Based on Shape Features. Journal of Agricultural Science and Technology 2012, 14, 755-765.

26. Delenne, C.; Rabatel, G.; Deshayes, M. An Automatized Frequency Analysis for Vine Plot Detection and Delineation in Remote Sensing. IEEE Geoscience and Remote Sensing Letters 2008, 5, 341-345.

27. Comba, L.; Gay, P.; Primicerio, J.; Ricauda Aimonino, D. Vineyard detection from unmanned aerial systems images. Computers and Electronics in Agriculture 2015, 114, 78-87.

28. Mancini, A.; Dyson, J.; Frontoni, E.; Zingaretti, P. Soil / crop segmentation from remotely sensed data acquired by Unmanned Aerial System. 2017 International Conference on Unmanned Aircraft Systems (ICUAS), 2017, pp. 1410-1417.

29. Khoshelham, K.; Nardinocchi, C.; Frontoni, E.; Mancini, A.; Zingaretti, P. Performance evaluation of automated approaches to building detection in multi-source aerial data. ISPRS Journal of Photogrammetry and Remote Sensing 2010, 65, 123-133. 
428 30. Mancini, A.; Frontoni, E.; Zingaretti, P. AUTOMATIC EXTRACTION OF URBAN OBJECTS FROM 429 MULTI-SOURCE AERIAL DATA. ISPRS - International Archives of the Photogrammetry, Remote Sensing and $430 \quad$ Spatial Information Sciences 2009, XXXVIII-3/W4, 13-18.

431 31. Mancini, A.; Frontoni, E.; Zingaretti, P.; Longhi, S. High-resolution mapping of river and estuary areas by 432 using unmanned aerial and surface platforms. 2015 International Conference on Unmanned Aircraft Systems, 433 ICUAS 2015 2015, pp. 534-542. 